\title{
Modelación Integrada de Sistemas Socio-ecológicos Complejos: Caso de Estudio la Ecorregión de la Mojana
} Integrated Modeling of Complex Socio-ecological Systems: Case Study of the Mojana ecoregion

Paula Andrea Villegas González ${ }^{1}$, Nathaly Triviño Leon ${ }^{1}$, Jorge Alberto Escobar Vargass ${ }^{1}$, Nelson Obregón Neira ${ }^{1}$, Mauricio González Méndez ${ }^{2}$ , Ramón Eduardo González Salazar ${ }^{1}$, María Juliana Flórez Flórez ${ }^{1}$

${ }^{1}$ Pontificia Universidad Javeriana. , ${ }^{2}$ Universidad Católica de Colombia.

Correo electrónico: pvillegas@javeriana.edu.co

Recibido: 20-11-2015. Modificado: 03-04-2016. Aceptado: 15-05-2016

\section{Resumen}

Contexto: Se ha observado que la modelación de sistemas socio-ecológicos complejos a partir de simulaciones basadas en agentes, tienen la ventaja de integrar diferentes procesos, escalas, variables y la posibilidad de generar escenarios con actores en el marco de procesos de modelación participativa. Método: Se diseña una herramienta computacional para la planificación y apoyo a los procesos de toma de decisión relacionados con la gestión de los recursos hídricos, específicamente para el caso de las inundaciones en la ecorregión de la Mojana (Colombia). Son desarrollados talleres de participación social relacionados con creencias, valores, redes sociales y resiliencia.

Resultados: El modelo tiene dos componentes: el primero representa la hidrodinámica de las inundaciones por medio de la plataforma numérica ISIS 2D; el segundo, en relación con los aspectos sociales de la región, se maneja a través de modelado de sistemas de agentes. Ambos sistemas están integrados en la plataforma NetLogo.

Conclusiones: La modelación integrada de sistemas socio-ecológicos complejos permite visualizar el comportamiento de la población y los recursos naturales en un territorio, contribuyendo al diseño de políticas y los procesos educativos con la participación de diferentes disciplinas y actores. Trabajos futuros, pueden enfocarse en la modelación regional y el análisis del impacto producido por el uso de estas herramientas.

Palabras clave: Modelación participativa, modelos basados en agentes, sistemas socio-ecológicos. Agradecimientos: A las comunidades de los municipios de Nechí (Departamento de AntioquiaColombia) y San Marcos (Departamento de Sucre-Colombia) por su participación en la investigación. A los grupos de investigación de Riesgo en sistemas naturales y antrópicos, de la Pontificia Universidad Javeriana y Gestión y tecnología para la sustentabilidad de las comunidades, de la Universidad Católica de Colombia.

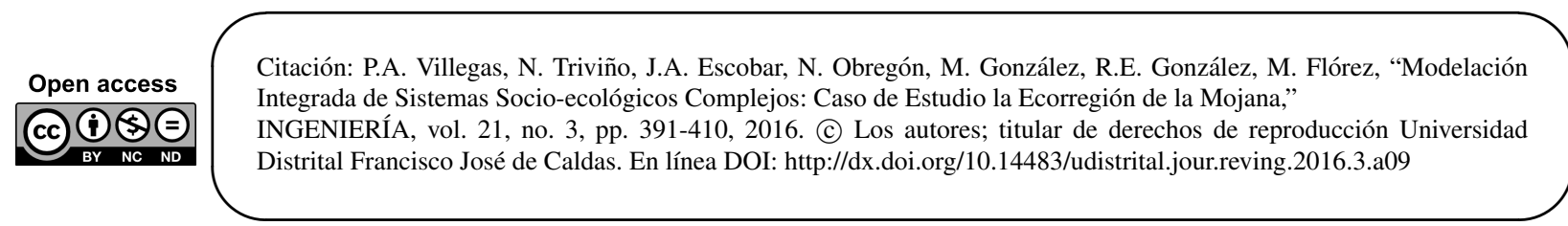




\begin{abstract}
Context: It was observed that the modeling of complex socio-ecological systems based on AgentBased Simulations has the advantage of allowing the integration of different processes, scales, variables and the possibility to generate scenarios with actors in the context of the modeling with stakeholders.

Method: A computational tool for planning and support of decision-making processes related with water resources management, specifi- cally in the case of floods in the Mojana ecoregion (Colombia) is designed along with social participation workshops related with beliefs, values, social networks and resilience.

Results: The model has two components: the first one represents the hydrodynamic of flooding by means of the numerical platform ISIS 2D. The second one, regarding the social aspects of the region, is handled via agent systems modeling. Both schemes are integrated into the NetLogo platform.

Conclusions: The integrated modeling of complex socio-ecological systems allow us to visualize the behavior of the population and the natural resources in a territory, contributing to the design of policies and educational processes involving different disciplines and actors. Future work will focus on regional modeling and the analysis of the impact produced by the use of these tools.

Keywords: Agent-based modeling, modeling with stakeholders, socio-ecological system.

Acknowledgment: Municipalities communities of Nechí (Antioquia-Colombia) and San Marcos (Sucre-Colombia) for their participation in the research. Researchers: Pontificia Universidad Javeriana's risk in natural and human systems group and Universidad Católica de Colombia's Management and technology for communal sustainability group.
\end{abstract}

\title{
1. Introducción
}

Durante los últimos años ha aumentado el uso de modelos caracterizados por la integración de múltiples métodos de modelación y tecnologías que hacen parte de varias disciplinas [1]. Dicho proceso ha surgido por la necesidad de representar la complejidad de los sistemas ecológicos, que para esta investigación considera elementos sociales y ambientales del territorio la Mojana.

La Mojana es una ecorregión de especial importancia para Colombia, que hace parte del complejo de humedales de la Depresión Momposina, la cual es una cuenca hidrográfica sedimentaria de $24.650 \mathrm{~km}^{2}$ reguladora de los caudales de los ríos Magdalena, Cauca y San Jorge. Tales humedales cumplen la función de amortiguación de inundaciones, facilitando la decantación y acumulación de sedimentos, funciones de control indispensables para la costa Caribe [2]. El ecosistema está siendo afectado por desequilibrios ambientales generados por el inadecuado uso, ocupación del territorio y por el inapropiado manejo de las cuencas de los ríos que allí confluyen; adicionalmente, la construcción de obras civiles ha generado daños en el equilibrio del complejo cenagoso y fluvial. En los últimos años la infraestructura se ha orientado al control del agua mediante obras que afectan la dinámica hídrica y desestabilizan los sistemas hidrobiológicos, en lugar de adaptarse a las condiciones del medio natural y social para su aprovechamiento. Las dinámicas ambientales y sociales, en conjunto, están generando peligros para la sostenibilidad de los valiosos ecosistemas naturales que la caracterizan y limitando el desarrollo socio-económico de la población allí asentada. A lo anterior se suma que es problemático que los procesos de toma de decisiones en algunas ocasiones se hacen desconociendo los diversos saberes y conocimientos locales de los habitantes, lo cual se 
agrava más aún cuando dichas decisiones se toman desde el gobierno centralizado que desconoce las dinámicas regionales. En este sentido, la investigación tiene como propósito representar este territorio como un sistema socio-ecológico complejo desde la ingeniería, vinculando elementos sociales y a través de la modelación basada en agentes.

Los ecosistemas son sistemas complejos [3], en los que patrones a niveles superiores surgen de interacciones y procesos generados en los niveles inferiores [4]. Las características clave de este tipo de sistemas son la no linealidad [3], la emergencia, la auto organización y su carácter impredecible [5]. Además, el concepto de escala, donde para cada nivel de resolución en el que el sistema es observado, se puede generar un modelo diferente para describir las características en esa escala [6]. Deben ser estudiados como un sistema compuesto de múltiples componentes que interactúan, cuyo comportamiento da lugar a funciones y estructuras agregadas [4]. La modelación integrada de sistemas complejos que propone esta investigación pretende apoyar los procesos de toma de decisión a escala local. Esto en el sentido que tiene la capacidad de representar mejor las dinámicas del sistema, explorar futuros posibles considerando diferentes escenarios [1] y es susceptible de entablar un diálogo que tome en cuenta los conocimientos locales de las comunidades.

En el análisis y modelación de sistemas ecológicos se han utilizado diferentes técnicas, con aproximaciones fundamentadas en las matemáticas, la física, la ecología o las ciencias sociales. Para el caso de la ecorregión de la Mojana, en 1977 la Misión Colombo Holandesa propone obras de infraestructura para la limpieza y adecuación de canales; en 1998 Corpoica (Corporación Colombiana de Investigación Agropecuaria) realiza una caracterización biofísica de la producción agropecuaria [30]; en el 2003 es propuesto un programa de desarrollo sostenible de la región de la Mojana que define un plan productivo, agropecuario y forestal [2]; en el 2006 con el Conpes 3421 el DNP (Departamento Nacional de Planeación) son definidas las estrategias para la reactivación económica y social de la Mojana; en el año 2011 es formulado el Plan de Ordenamiento Ambiental y Desarrollo Territorial propone una modelación matemática hidráulica y de calidad de agua. Actualmente, el Fondo de Adaptación realiza estudios para la intervención integral en la reducción del riesgo de inundación [32]. Como se observa, todas estas iniciativas acuden a diferentes ámbitos de saber; sin embargo, en ninguna de ellas la participación de las comunidades locales ha jugado un rol protagónico. Esta última característica ha generado que decisiones, por ejemplo, en inversión de infraestructura, no se hayan podido concluir por la resistencia de las comunidades. Esta investigación, desde un enfoque interdisciplinar y orientada por el diálogo de saberes, busca lograr una modelación integrada teniendo en cuenta que la multidimensionalidad de los problemas exige ir más allá de la disciplina de la ingeniería hidráulica. Por ejemplo, de la física (dado que el agua es uno de los componentes estructurales del territorio) o de las ciencias sociales (para considerar las dinámicas sociales y comunitarias como otros determinantes de las dinámicas territoriales).

Remolina (2014) [45] entiende la interdisciplinariedad "como el paso de la multiplicidad de las disciplinas a su integración en un pensamiento complejo" y él enfatiza en tres niveles. El primero, la uni-disciplinariedad y multidisciplinariedad que consiste en el estudio de un mismo objeto por varias disciplinas. El segundo, Inter-disciplinariedad que implica la transferencia de métodos de una disciplina a otra. El tercero, la trans-disciplinariedad que se basa en la existencia de diversos niveles de realidad, entre los cuales se da discontinuidad, con saltos cualitativos entre uno y otro nivel. De esta manera, "la interdisciplinariedad busca restituirle a la realidad su integridad recons- 
truyéndola en su complejidad por medio de la integración de los conocimientos". Pero, advierte, no todo es integrable inmediatamente dado que la complejidad de la realidad es orgánica y en un organismo cada dimensión o componente tiene funciones y conectores diferentes.

En el proceso interdisciplinar que se quiere abordar en esta investigación, las diversas disciplinas han de integrarse de manera orgánica y gradual. Aquí se dan los primeros pasos para pasar del primer nivel de interdisciplinariedad al segundo, pues se asume que no es suficiente el abordaje del territorio desde la Ingeniería hidráulica, sino que debe empezar a contemplar aportes de las ciencias sociales para entrar en diálogo con los conocimientos locales. En esta línea, es pertinente el énfasis de Amozurrutia (2011) [45] en dos señalamientos que son fundamentales para esta investigación: primero, que "es necesario hacer un trabajo interdisciplinario que permita enfrentar lo complejo de nuestra realidad social en la multidimensionalidad de sus problemas"; esta complejidad se ve reflejada en las características de la ecorregión; segundo que es crucial promover "una forma de investigación cimentada en lenguajes comunes, como la epistemología y las matemáticas, y potenciada por un pensamiento sistémico que use recursos computacionales como plataforma generativa de conocimiento". En este caso, el proceso de investigación apunta a la construcción de lenguajes comunes, un pensamiento sistémico para la solución de problemas y el diseño recursos computacionales como herramientas para la simulación.

Varias publicaciones se han destacado en este tema, los primeros trabajos en modelación de uso de la tierra y cambio en la cobertura vegetal son presentados por Parker (2012) [7], y se llegó a la conclusión de que ha sido una de las principales aproximaciones para representar la complejidad, encontrando las propiedades emergentes definidas como "un resultado macroscópico como resultado de las sinergias e interdepencias entre los componentes del sistema inferior" [7]. Bousquet and Le Page (2004) [8], hacen una revisión de las simulaciones multi- agentes (MAS) para la gestión de los ecosistemas, donde se concluye que esta técnica de modelación es útil para los problemas de integración de los aspectos sociales y espaciales. Matthews et al., (2007) [9] presentan una revisión relacionada con la manera de incorporar la toma de decisiones sobre el uso del suelo a través de la modelación. Para esto tienen en cuenta modelos basados en agentes de (i) análisis de políticas y planificación, (ii) modelos participativos, (iii) Explicación de patrones espaciales del uso de la tierra, entre otros. Concluyen que la modelación basada en agentes, tiene la ventaja de incorporar procesos sociales y enlazar dinámicamente estos procesos y los ambientales. An (2012) [10] hace una revisión sobre los sistemas humanos y naturales acoplados, a la luz de la teoría de la complejidad y sus aplicaciones, donde resalta la importancia de generar protocolos o arquitecturas genéricas, que puedan servir como puntos de referencia, que incluyen recomendaciones sobre la estructura de los modelos, la elección de los mismos y los elementos claves en el modelado de las decisiones humanas.

Diferentes temas de aplicaciones ambientales han sido investigados en: Balbi et al., (2013) [11] donde se representan los efectos del cambio climático. Brown et al., (2004) [12] donde se realizan modelos de desarrollo residencial en la periferia rural-urbana. Barreteau and Bousquet (2000) [13] acerca de la viabilidad de los sistemas de riego y la influencia de las redes sociales. Schreinemachers and Berger (2011) [14], para la toma de decisiones en procesos de explotación agrícola. Balbi et al., (2015) [15] sobre los servicios ecosistémicos relacionados con los rendimientos del cultivo, suministro de agua y la calidad, el clima y la calidad del aire. Anselme et al., (2010) [16], sobre 
biodiversidad y conservación, donde se construyó una representación compartida del territorio y desarrollaron escenarios de gestión alternativos con actores locales.

Por todo lo expresado con anterioridad, se puede concluir que la modelación de sistemas socioecológicos complejos a partir de simulaciones basadas en agentes, tiene la ventaja de poder integrar diferentes procesos, escalas, variables y la posibilidad de generar escenarios con actores en el marco de procesos de modelación participativa. Esto se convierte en una potencialidad para los procesos interdisciplinarios de planeación e intervención en un territorio. Se han encontrado tres retos en la aplicación de la simulación basadas en agentes que se esperan abordar en esta investigación, sin expresar que ya están resueltos: (i) modelación de la conducta de los agentes. (ii) análisis de sensibilidad, verificación y validación y el (iii) acoplamiento de modelos socio-demográficos, ecológicos y biofísicos [41].

En este artículo son presentados algunos avances conceptuales, metodológicos y resultados del modelado apoyado en agentes en el municipio de Nechí (Departamento de Antioquia-Colombia), que permiten soportar los procesos de toma de decisión.

\section{Materiales y métodos}

\subsection{El diseño y la modelación en ingeniería}

Esta investigación es abordada desde el marco conceptual del diseño en ingeniería, con un componente social y de participación que generalmente no son abordados desde la ingeniería clásica. Según Kroes (2012) [17], el diseño "es un proceso de invención, una generación inteligente y evaluación de especificaciones de nuevos objetos, artefactos; cuya forma y función logren los objetivos establecidos y satisfagan las necesidades, pero también contemplen limitaciones y restricciones".

El diseño tiene una actividad mental y una actividad física. Respecto al aspecto mental "El verdadero trabajo de fabricación se realiza bajo la guía de un modelo, de acuerdo con el cual se construye el objeto. Dicho modelo puede ser una imagen contemplada por la mente o bien un boceto en el que la imagen tenga ya un intento de materialización mediante el trabajo" [18].

El modelo conceptual utilizado en esta investigación es presentado en la Figura 1. Allí se observa que el componente principal es el territorio concebido como "espacio construido por los grupos sociales a través del tiempo, a la medida y a la manera de sus tradiciones, pensamientos, sueños y necesidades, territorios que significan mucho más que espacio físico poblado por distintas formas de vida que se relacionan, cooperan y compiten entre sí; lo que permite concebir al territorio como un campo relacional" [21]. El cual está compuesto por los niveles del agua, los usos del suelo, la población y las relaciones entre estos. Estos tres componentes y los procesos de modelación participativa constituyen el artefacto para el modelado integrado de sistemas socio-ecológicos complejos.

La modelación participativa pretende incorporar elementos del ecosistema y a la población conformada por grupos de seres humanos. Que no solo se encuentran representados o imaginados en la herramienta de simulación, sino también en el proceso de diseño, construcción e implementación del modelo. Para modelar las dinámicas sociales, el uso de reglas que representan la conducta de los 
agentes es un reto y los conceptos de la propuesta socioeconómica inspirada en la teoría evolutiva pueden ser utilizados [47]. De tal manera que puedan integrarse los flujos de relación entre diferen-

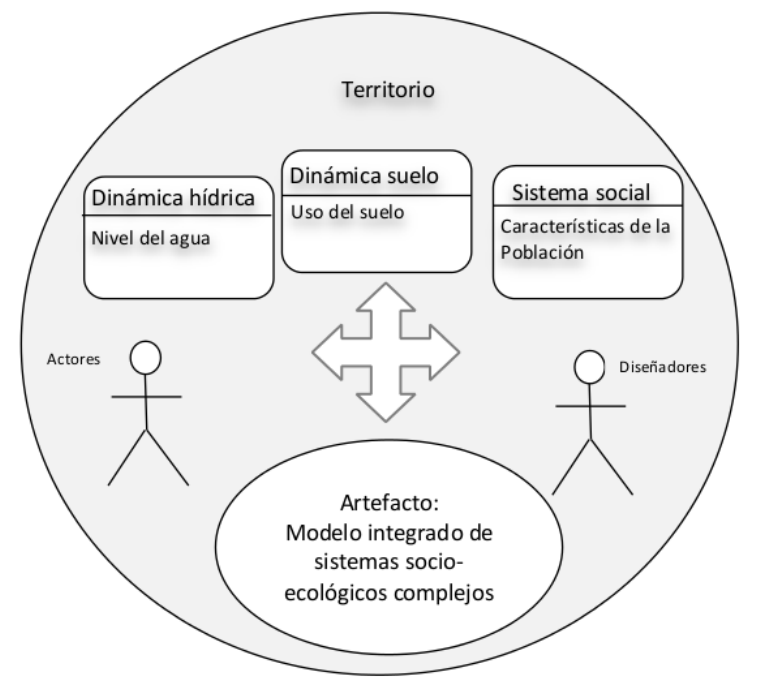

Figura 1. Modelo conceptual utilizado para este estudio. Los rectángulos representan los submodelos que integrados conforman el artefacto, un modelo integrado de sistemas híbridos complejos. tes submodelos. Todas las entidades, agentes y recursos asociados pueden ser concebidos como portadores de reglas y estas reglas pueden tener una trayectoria que pasa por el origen, adopción y retención. Pero además son analizadas utilizando los niveles micro, meso y macro que tienen relación directa con la trayectoria de la regla. En este sentido, se pretenden incluir reglas a diferentes niveles, micro, meso y macro, en el marco la herramienta de simulación. De tal manera que se puedan generar eventos hipotéticos, comúnmente denominados escenarios [48]. $\mathrm{El}$ artefacto es una herramienta para explorar futuros y posibles estrategias para implementar en un territorio, producto de un trabajo interdisciplinar con la participación de las comunidades locales.

Según Epstein (2008) [19] "Cualquiera que se aventure a proyectar, o imaginar una dinámica" está ejecutando un modelo. Los modelos pueden ser predictivos o explicativos (Epstein, 2008). Además de predecir pueden ser utilizados para explicar, descubrir nuevas preguntas y educar al público en general. El propósito del modelo presentado responde a ¿cómo se integran los resultados de modelación hídrica con elementos sociales, que permita contribuir al entendimiento de las dinámicas del territorio y la generación de escenarios? Adicionalmente, los modelos pueden clasificarse como implícitos o explícitos [19]. El modelo presentado es implícito, dado que los supuestos están ocultos, su consistencia no se ha probado, sus consecuencias lógicas y su relación a los datos es desconocida. Se busca el diseño de un modelo formal que según Schwaninger (2010) [20], tiene entre sus características, la generación de modelos de simulación donde se pueden llevar a cabo experimentos con el fin de obtener el conocimiento en el sistema real estudiado.

\subsection{Modelación integrada}

Desde diferentes saberes, disciplinas y específicamente la ingeniería existen diversas alternativas para entender dinámicas en un territorio. Los modelos físicos (hidrológico, hidráulico, hidrodinámico, de agua subterránea, etc.) se utilizan para predecir el estado espacial y temporal de los recursos hídricos [22]. Los modelos demográficos pueden simular aspectos socioeconómicos, como proyecciones de la demanda del sector social, pronóstico de población por edad y nivel educativo, entre otros [23]. Los modelos de gestión pueden ser concebidos para ayudar a las organizaciones a completar sus tareas con eficacia [24]. Sin embargo, implementar alguno de estos podría no ser suficiente para representar las dinámicas en el territorio. Por lo tanto, en este estudio se requiere explorar el diseño de un modelo, que como afirman Letcher et al., (2013) [25] permita tener una comprensión integrada de los procesos donde se tengan en cuenta los componentes físicos y sociales, sus interacciones complejas, cómo responden a diferentes cambios, además del conocimiento 
y opinión de las partes interesadas.

El enfoque se plantea integrado en la medida que la región de la Mojana es abordada como un territorio, un sistema complejo [17], donde están involucrados: objetos, naturaleza, artefactos técnicos, actores humanos, entidades sociales, normas y leyes. Son presentados fundamentos de la modelación integrada según Letcher et al., (2013) [25] con las ideas que han surgido en la investigación:

- El enfoque integrado surge porque la gestión de los recursos naturales tiene impactos en otros aspectos como: sociales, económicos, ambientales y de infraestructura. La integración es parte de un enfoque sistémico, donde aparecen varias dimensiones y dinámicas del territorio como un todo.

- La modelación integrada es un proceso donde también aparecen integrados los actores a través de procesos de modelación participativa. Estos pretenden: (i) incrementar el conocimiento de los actores, (ii) lograr el entendimiento de un sistema y sus dinámicas bajo varias condiciones como aprendizaje colaborativo e (iii) identificar y clarificar los impactos de situaciones dadas soportando la toma de decisiones [26]. Resulta necesario contar con la integración y conocimiento de los actores en las diferentes etapas de modelación, no solo para empoderarlos de la herramienta o usarla sino también para generar un proceso legitimación. La integración de disciplinas o saberes también se contempla en los procesos de modelación integrada. El objetivo es lograr transformar esta información en conocimiento al interior del modelo y tener en cuenta el conocimiento de dichos expertos.

- La integración de escalas. Los componentes de un sistema pueden operar a diferentes escalas espaciales y temporales. Para examinar aspectos de hidrología los límites de las cuencas pueden ser los más apropiados, sin embargo, cuando se consideran aspectos sociales y económicos, estas escalas pueden variar a hogares, granjas, instituciones grupos sociales, o entidades. La selección de la escala tiene en cuenta (i) la escala de interés para los usuarios o actores interesados, (ii) la escala a la que los procesos pueden ser representados, (iii) el vínculo entre los componentes del modelo que representan los procesos en diferentes escalas y (iv) las limitaciones prácticas tales como datos, capacidad computacional, cubrimiento de la población en términos de conocimiento experto. En la Tabla I se presenta un ejemplo, donde se puede observar un análisis de escalas temporales y espaciales para la ecorregión de la Mojana.

- La integración de procesos resulta importante, en la medida que se deben combinar dos o más procesos en el territorio que responden a los componentes a modelar. La modelación puede llegar a requerir la combinación de técnicas de modelación de diferentes disciplinas.

\subsection{Descripción del modelo}

En los últimos años se ha avanzado en el desarrollo de herramientas computacionales para la planificación y apoyo a los procesos de toma de decisión en la gestión del recurso hídrico. Los modelos integrados han sido fundamentales en la captura de la complejidad de los usos del agua [28]. 
Tabla I. Análisis de las escalas en el territorio: ecorregión de la Mojana

\begin{tabular}{|c|c|c|}
\hline \multicolumn{3}{|c|}{ Elementos que integran el sistema que se quiere modelar } \\
\hline Inundación & Población & Usos de suelo \\
\hline $\begin{array}{l}\text { El análisis espacial: se pueden observar y } \\
\text { analizar las manchas de inundación en la } \\
\text { cuenca Magdalena Cauca, o en el núcleo } \\
\text { de la región de la Mojana, dada por la va- } \\
\text { riación de los niveles del agua. También } \\
\text { se puede observar la variación de los ni- } \\
\text { veles del río o de la ciénaga. }\end{array}$ & $\begin{array}{l}\text { El análisis espacial: los satisfactores de las } \\
\text { necesidades básicas de los seres humanos } \\
\text { pueden cambiar. Por ejemplo: para la nece- } \\
\text { sidad de subsistencia. Los satisfactores co- } \\
\text { rrespondiente a la alimentación, el trabajo } \\
\text { y la salud durante el periodo de inundación } \\
\text { pueden cambiar [27]. Esto podría analizar- } \\
\text { se desde la perspectiva de la persona, de las } \\
\text { personas que conforman el barrio, las fami- } \\
\text { lias, de los barrios que conforman el muni- } \\
\text { cipio, de una comunidad afín como por ejem- } \\
\text { plo de mujeres cabeza de familia, entre otras. }\end{array}$ & $\begin{array}{l}\text { El análisis espacial: en la parcela, en la vivienda, } \\
\text { en el municipio, en el departamento, en el núcleo } \\
\text { de la región de la Mojana, las características y el } \\
\text { uso del suelo puede cambiar.Obras de infraestruc- } \\
\text { tura pueden construirse, pueden dañarse, pueden } \\
\text { estar en periodos de mantenimiento. Estas pueden } \\
\text { ser en una vía, en una sección del río, etc. }\end{array}$ \\
\hline $\begin{array}{l}\text { El análisis temporal:mensual, anual, } \\
\text { horario,diario. }\end{array}$ & $\begin{array}{l}\text { El análisis temporal: una vida (nacimiento- } \\
\text { muerte), una etapa de la vida (niñez, juven- } \\
\text { tud),los procesos políticos (un periodo de go- } \\
\text { bierno), escalas demográficas, adolescencia. } \\
\text { Una hora, un día, un mes, un año. }\end{array}$ & $\begin{array}{l}\text { El análisis temporal: duración de la cosecha, dura- } \\
\text { ción de siembra, tiempo de vida útil de la obra de } \\
\text { infraestructura.Una hora, un día, un mes, un año. }\end{array}$ \\
\hline
\end{tabular}

En este sentido es necesario, para el caso de la ecorregión de la Mojana, contar con modelos que permitan simular grupos autónomos como asentamientos poblacionales o individuos y sus interacciones entre sí y con el territorio. Por tal motivo se ha seleccionado la técnica de simulación basada en agentes, dado que tiene la capacidad de acoplar los procesos de inundación, con el territorio y las dinámicas sociales. A continuación, se describen algunos aspectos para la construcción e implementación del modelo integrado con el soporte conceptual de los trabajos de Letcher et al., (2013) [25] y Refsgaard et al., (2007) [29]. Primero se hace una descripción de la zona de estudio y luego es presentada la arquitectura del modelo.

\subsubsection{Descripción de la zona de estudio}

La ecorregión de la Mojana está ubicada entre la región Andina y las llanuras del Caribe, esta es de gran importancia ya que hace parte de la subregión fisiográfica conocida como Depresión Momposina, la cual está limitada por el río Cauca al oriente, el río San Jorge al occidente, el Brazo de Loba del río Magdalena al norte y las estribaciones de las Serranías de Ayapel y San Lucas al sur [30].

Los primeros pobladores fueron los indígenas Zenues, quienes colonizaron y adecuaron la depresión Momposina e iniciaron su poblamiento desde el siglo IX a.C, hasta los siglos X-XII d.C [49]. En este proceso de poblamiento aparecen ingenieros hidráulicos tradicionales muy importantes que marcaron la historia del manejo de los recursos hídricos en Colombia y en Latinoamérica. Con el fin de adaptarse a los periodos de inundación construyeron plataformas artificiales (de entre 2 o $3 \mathrm{~m}$ de altura) para instaurar sus viviendas sin que los niveles del agua las afectara. Este sistema estaba compuesto por canales artificiales y camellones (partes elevadas entre canal y canal) que cumplían la doble función de encausar el agua hacía el mar y servir de lugar de cultivo. Las aguas se distribuían de manera uniforme, circulaban más lentamente durante la época de inundaciones y, en época de sequía, permanecían humedeciendo los camellones [49]. Dichas técnicas fueron abandonadas y las causas no han sido establecidas. Aunque, este abandono se le atribuye a fenómenos ambientales (periodos de sequías), muy probablemente fue producto de la ola de colonización española y sus efectos de supresión cultural y epistémica, en este caso, de los avanzados conocimientos hidráuli- 
cos del momento. En todo caso, uno de las características especiales del proceso de poblamiento español que empezó en el siglo XVI fue la introducción de ganado en la zona por parte de los hermanos Heredia. De esta forma se instaló en el territorio la ganadería y explotación agrícola extensiva. Los hechos del proceso de colonización marcaron una nueva historia para la región. Las actividades económicas, ya no vistas como procesos de subsistencia de sus habitantes empiezan a enfocarse en la sobreexplotación del suelo, el agua y los seres humanos.

Actualmente, en épocas de invierno la región tiende a inundarse en casi toda su extensión y es por esta razón que el gobierno colombiano, a fin de controlar estos desastres construye a partir del CONPES 3421 de 2006 (Consejo Nacional de Política Económica y Social-Colombia) obras de infraestructura como compuertas, canales de drenaje y riego, estructuras de control hidráulico y algunos terraplenes o diques, para evitar desastres a gran escala. Entre ellas se encuentra un dique marginal multipropósito [31], que se encuentra localizado desde Colorado (Antioquia) hasta Achí (Bolívar) por todo el margen izquierdo del río Cauca. Este dique, durante el fenómeno de la niña de los años 2010-2011 presentó gran cantidad de fallas en las zonas más débiles (Nuevo Mundo, Pedro Ignacio y Santa Anita, Figura 2), debido al aumento de la creciente del río Cauca, generando inundaciones en una gran parte de la región. Entre los municipios más afectados se encuentra Nechí, con una población de 23.502 habitantes, en un territorio de $914 \mathrm{Km}^{2}$ (censo en Colombia del año 2011). Este territorio cuenta con una gran riqueza hídrica, ecológica y agropecuaria, pero debido a su localización está expuesto a frecuentes inundaciones. Esta situación ocasiona que el municipio no tenga un satisfactorio desarrollo económico, además causa enfermedades que afectan principalmente a niños y adultos mayores [31]. Según el DANE (Departamento Administrativo Nacional de Estadística), las Necesidades Básicas Insatisfechas alcanzan el 68.13\% (2011), el Índice de Desempeño Integral 44.99\% (DNP, 2013), y el GINI de tierras 0.627 (UniAndes, IGAC y U de Antioquia, 2012). Dichas características hacen evidente la dependencia que tiene la población de los ríos desde el punto de vista económico y social. Por lo que resulta importante analizar el comportamiento de las dinámicas entre el uso del suelo, la población y el recurso hídrico. Los resultados del ejercicio de modelación podrían generar herramientas en el marco de la gestión de los recursos naturales en el municipio, además de fortalecer los procesos de planeación. Esto contribuiría a los procesos del manejo integral de la Cuenca.

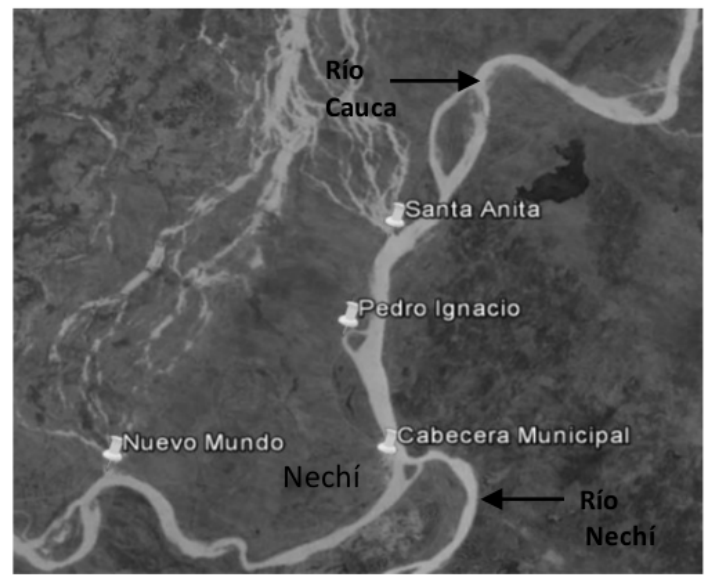

Figura 2. Localización del Municipio de Nechí (Antioquia) y zonas del dique Marginal que han presentado fallas: Santa Anita, Pedro Ignacio y Nuevo Mundo. Imagen obtenida de Google Earth.

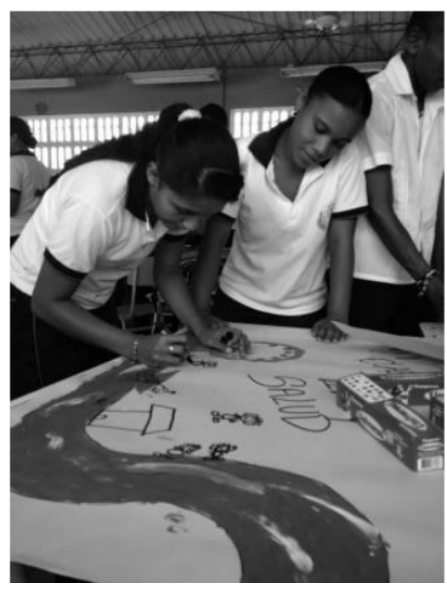

Figura 3. Trabajo con las comunidades en la ecorregión de la Mojana. Significado del agua para la Población. 


\subsubsection{Trabajo con las comunidades}

Esta idea de investigación surge entre los años 2011-2012, en el marco del trabajo con las comunidades locales de la Mojana emprendido como parte del equipo encargado de la formulación del Plan de Ordenamiento Ambiental y Desarrollo Territorial bajo el acuerdo con los once alcaldes municipales (autoridades locales) y con el fin de formular líneas estratégicas para varios temas, entre los más determinantes, el del manejo del recurso hídrico.

Posteriormente entre 2013 y 2015, se llevaron a cabo dos visitas a la Mojana, específicamente en los municipios de San Marcos y Nechí. En San Marcos se desarrollaron tres talleres con las instituciones educativas. El primero sobre creencias y valores y cuyo objetivo fue elaborar un diagnóstico participativo para profundizar sobre los metarelatos religiosos, las creencias populares, costumbres y valores de la población (Figura 3). El segundo, un taller de redes sociales e identidad cuyo propósito fue evaluar desde la experiencia comunitaria, elementos que ayudaran a esbozar características de la identidad local y regional, así como a vislumbrar los sueños compartidos. Por último, en el tercer taller sobre resiliencia tuvo como objetivo reconocer las cualidades y fortalezas que permiten a las personas enfrentar positivamente situaciones desfavorables. El objetivo de estos tres ejercicios tanto en San Marcos como en Nechí fue explorar los sentidos del agua, las experiencias con construcciones palafíticas en las escuelas, pero además identificar variables que debían ser incluidas en el modelo. Para el desarrollo de los talleres se acudieron a variadas técnicas de investigación de corte cualitativo; se hicieron cartografías sociales con niños y jóvenes, conversatorios comunitarios con profesores y padres y madres de familia. Además, se realizaron recorridos territoriales durante los cuales se mantuvieron conversaciones informales con los pobladores. De esta manera surge un acuerdo para iniciar un proceso de formación comunitaria en modelación que permita fortalecer los procesos de toma de decisiones en los municipios. La pertinencia de esta formación se justifica en graves hechos precedentes. Por ejemplo, en el caso del municipio de Nechí se perdió un programa de construcción de viviendas, dado que no se conocían con claridad los niveles que podría alcanzar el agua en la zona en periodos de inundación, por lo que la comunidad considera que comprender la relación entre las dinámicas hídricas y elementos sociales puede apoyar el conocimiento del territorio y la posterior toma de decisiones. Como resultado de estos ejercicios se identificó conjuntamente con la población local, una serie de variables iniciales que apoyaron la construcción del modelo presentado en esta investigación. Una vez la herramienta sea implementada se hará un ejercicio adicional de formación y validación con las comunidades locales, que además le otorgue sentido y legitimación al proceso de modo que pueda ser apropiado por la población.

\subsubsection{Arquitectura del modelo}

En esta sección se presenta con detalle los elementos del modelo y la metodología para generar el proceso de modelación integrada en la ecorregión de la Mojana. En la Figura 4 se presenta la estructura del modelo. La capa superior es el modelo de agentes que representa los habitantes, la del medio corresponde a las características de uso del suelo y la inferior describe el proceso de inundación. El suelo se pretende modelar como un autómata celular [32] cuyas celdas albergan una cantidad de la riqueza de la unidad territorial, medida no solo en términos de recursos naturales, sino también en elementos humanos como infraestructura y servicios, entre otros. Tiene capacidad 


\section{Capas}

Población

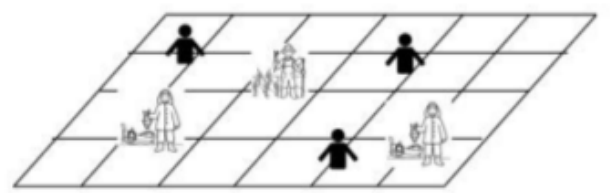

Uso del suelo

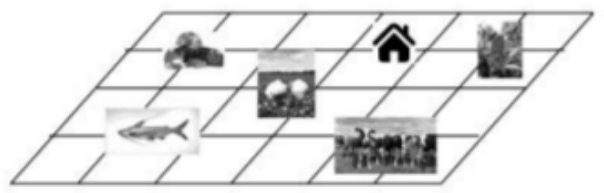

Nivel y velocidad del agua

\section{Submodelos}

Sistema de agentes que toma provecho del territorio. Dependiendo de la adaptación del agente al agua, esta degrada o no su bienestar.

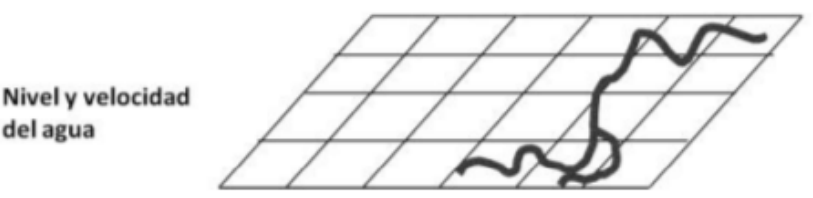

Automata celular cuyas celdas albergan cantidad de grano: riqueza de la unidad territorial.

territorial. Modelo hidrodinámico ISIS 20. El nivel del agua puede ser atractivo o repulsivo para los agentes. $\boldsymbol{E l}$ agua es un agente: ente gobernado por reglas.

Figura 4. Componentes del modelo integrado [32]. Las capas propuestas están inspiradas por Berger et al. , (2006) [28], mientras que los sub-modelos toman como base a los aplicativos: NetLogo wealth distribution model (Wilensky, 1998, 1999) [34], NetLogo Urban Suite-Sprawl Effect model (Felsen and Wilensky, 2007; Wilensky, 1999) [35] y NetLogo Erosion model (Dunham et al ., 2004; Wilensky, 1999) [36].

de regeneración por lo tanto la cantidad de riqueza que se consuma puede ser recuperada en el tiempo [32]. Los autómatas celulares son reconocidos como modelos computacionales sencillos, cuyo espacio es un arreglo de celdas donde agentes y reglas evolucionan en el tiempo. Estos son usados para modelar la complejidad de la naturaleza [52] y en este caso la evolución de la riqueza en el suelo.

Las poblaciones humanas (La capa de población) están representadas por un sistema de agentes que toman provecho del suelo para mejorar sus ingresos, sin embargo, la no adaptación a las condiciones de inundación puede degradarla. La interacción de los agentes con el suelo conlleva al mejoramiento o empobrecimiento del mismo. Con los niveles del agua (la capa nivel y velocidad del agua) arrojados por un modelo hidrodinámico (plataforma ISIS 2D), se simula una inundación que se produce periódicamente [33]. El nivel de agua en las unidades territoriales puede ser atractivo o repulsivo para los agentes (dependiendo del su nivel de adaptación en zonas con inundación y secas). La presencia periódica de la inundación, la capacidad de regeneración y modificación del territorio, sumadas a la posibilidad de cambio en los agentes hacen que el territorio se caracterice por exhibir un entorno variante en el tiempo [32].

\section{- Modelación hidrodinámica:}

Para la modelación hidrodinámica se utilizó la plataforma ISIS 2D [37]. En este caso se propone usar en la modelación del proceso de inundación de los ríos Cauca y Nechí. Esta herramienta permite conocer las condiciones hidrodinámicas del cuerpo de agua como: velocidad y elevación del nivel del agua. Las suposiciones utilizadas son: (i) el fluido es homogéneo, (ii) las velocidades verticales son mucho menores que las horizontales, (iii) las aceleraciones verticales pueden des- 
preciarse, (iv) la distribución de presiones es hidrostática y (v) las fuerzas inerciales son mayores que las viscosas. Teniendo en cuenta las suposiciones presentadas, el software resuelve las ecuaciones de aguas someras por medio del método de diferencias finitas en el espacio y los métodos numéricos utilizados para la discretización temporal son: ADI, TVD, FAST y FAST Dynamic [37].

Estas se derivan de las ecuaciones de NavierStokes para flujo incompresible. El sistema de ecuaciones consta de las ecuaciones de movimiento horizontal y la ecuación de continuidad. El conjunto de ecuaciones diferenciales parciales en combinación con condiciones iniciales y de frontera se resuelven en una cuadrícula de diferencias finitas. En la Figura 5 se observa el esquema utilizado para la formulación de las ecuaciones de aguas someras que resuelve ISIS 2D.

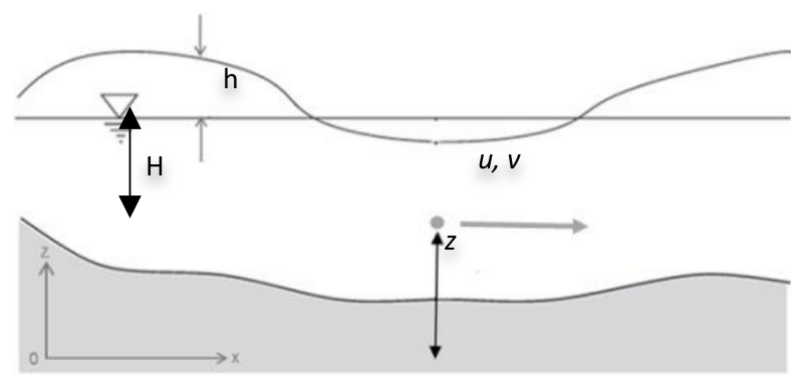

Figura 5. Representación de las ecuaciones de aguas someras. Wirasaet et al. , (2014) [38].

Donde $H$ es la profundidad promedio del agua, $h$ la perturbación de la superficie libre y $u, v$ las velocidades horizontales, $g$ la gravedad, $S_{x}$ y $S_{y}$ representan los términos fuente (fricción, viento coriolis, etc). A continuación, se muestra la forma general de las ecuaciones de aguas someras. La ecuaciones (1)(2) representan las ecuaciones de momento en las direcciones $x$ y $y$. La ecuación (3) representa la conservación de masa [39].

$$
\begin{aligned}
& \frac{\partial u}{\partial t}+u \frac{\partial u}{\partial x}+v \frac{\partial u}{\partial y}+z(u, v)=-g \frac{\partial h}{\partial x}+S_{x} \\
& \frac{\partial v}{\partial t}+u \frac{\partial v}{\partial x}+v \frac{\partial v}{\partial y}+z(u, v)=-g \frac{\partial h}{\partial y}+S_{y} \\
& \frac{\partial h}{\partial t}+\frac{\partial u}{\partial x}(H+h)+\frac{\partial}{\partial y}[(H+h) v]=0
\end{aligned}
$$

Para la selección de las condiciones iniciales y de frontera se hizo una encuesta con las comunidades que se ubican en las orillas del río y en los lugares donde se han generado rompimientos de obras de infraestructura. Allí se indaga sobre los niveles que alcanza el agua en diferentes épocas del año, la relación del agua con las actividades diarias y los impactos que se producen durante los periodos de inundación.

\section{- Modelación de la población:}

Se consideran dos tipos de agentes que representan el comportamiento de la población en la Mojana: agrícolas y anfibios que a su vez pueden adoptar dos estados: buscadores y asentados. Los atributos de los agentes se resumen en la Tabla II. Al comienzo el sistema inicializa el número total de agentes (agrícolas y anfibios); para cada uno de los cuales las rutinas son las mismas por población (i.e. buscar, moverse, asentarse, reproducirse y morir). La principal diferencia entre los dos tipos de agentes consiste en que el proceso de asentarse depende de la condición del suelo (i.e. seco o inundado). Las rutinas están predefinidas en función de la riqueza de las parcelas, no 
obstante su incremento y estabilidad dependen adicionalmente de la velocidad de búsqueda (i.e speed) y de la riqueza acumulada.

Tabla II. Atributos de la población en el modelo [32].

\begin{tabular}{ll}
\hline \multicolumn{1}{c}{ Atributo } & \multicolumn{1}{c}{ Descripción } \\
\hline Age & Edad del agente \\
\hline Wealth & Cantidad de riqueza que un agente tiene \\
\hline Lfe-expectancy & Máxima edad que un agente puede llegar a tener \\
\hline Metabolism & Cantidad de riqueza que un agente puede llegar a consumir \\
\hline Stay-counter & Tiempo de establecimiento de un agente en una parcela \\
\hline Patience-counter & Tiempo de búsqueda de un agente \\
\hline Speed & Velocidad del agente \\
\hline
\end{tabular}

Asimismo, estás últimas dan lugar a la configuración de tres clases sociales alta, media y baja (i.e. High, Medium, Low). Cuando el tiempo de búsqueda de un agente se agota, este puede cambiar su condición de anfibio a agrícola o viceversa [32]. El agotamiento es función de presiones selectivas del ambiente sobre el agente. Con estas condiciones se calcula el coeficiente de GINI que representa la desigualdad en el territorio; pero además se puede analizar el nivel de vulnerabilidad (en el marco de la gestión del riesgo) de la población frente al fenómeno de la inundación.

\section{- Modelo con sistemas de agentes:}

Los agentes son un sistema autónomo y proactivo capaz de interactuar con otros agentes para satisfacer sus objetivos. Entre las características principales de los agentes se encuentra que son: autónomos, proactivos, situados y dirigidos por entidades de software [40]. En el marco de la modelación integrada, los sistemas de agentes permiten la simulación de los muchos futuros posibles emergentes de sistemas complejos, son herramientas de apoyo para la toma de decisión y ofrecen a los gestores de recursos naturales con la capacidad de comprender mejor la dinámica actual de su sistema [1]. En la Tabla III son presentadas posibles variables y reglas a usar a futuro en el modelo de agentes para el municipio de Nechí. La participación de los actores durante el proceso de modelación ha resultado fundamental, de tal manera que (i) se logra comprender el funcionamiento de la herramienta, (ii) los actores pueden contribuir a la generación de variables y reglas (ii) la herramienta puede ser ajustada según la visión de los actores y se logra la (iii) generación de esce-

Tabla III. Posibles variables del modelo Elementos que integran el sistema que se quiere modelar

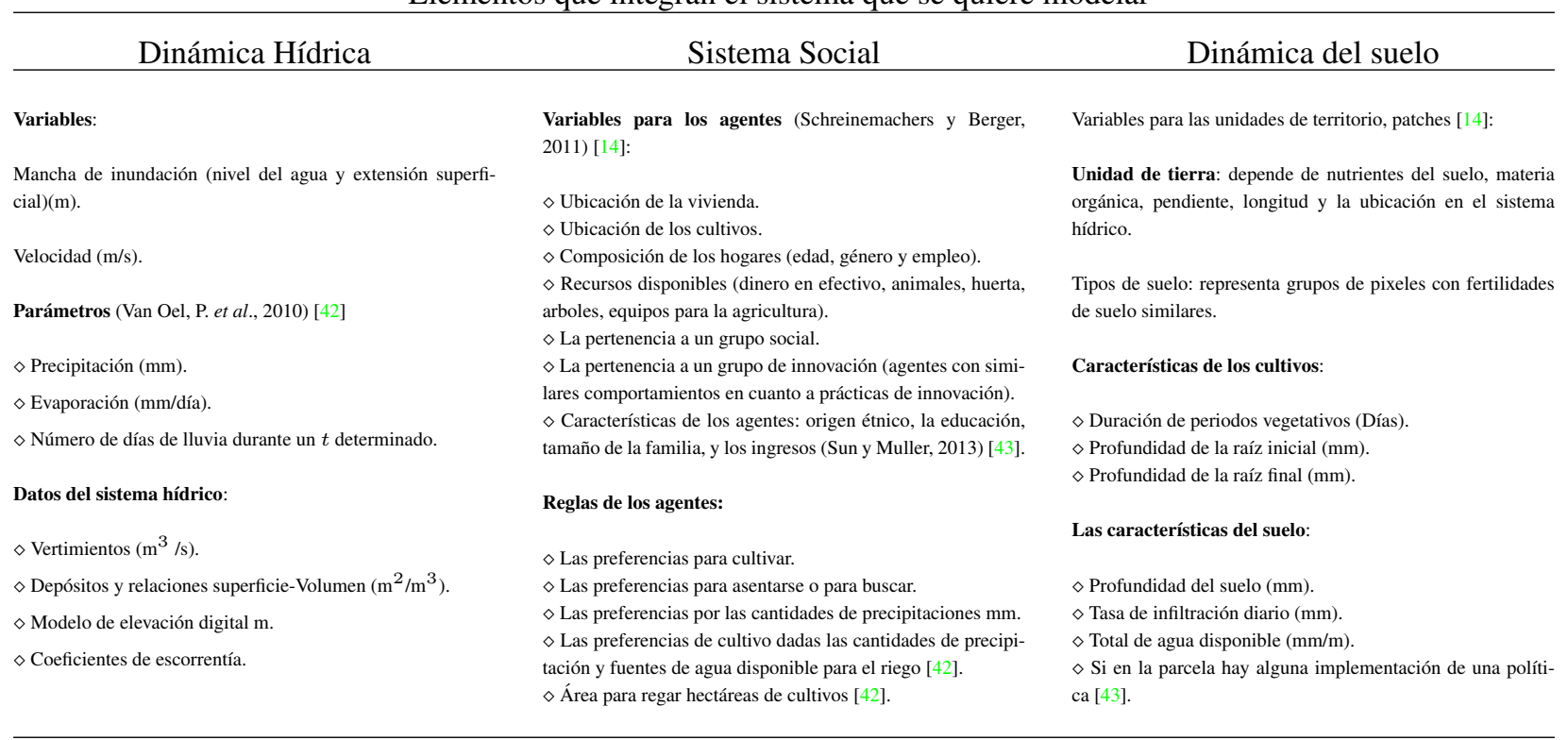


narios. Además, se considera como parte fundamental del proceso de modelación participativa el rediseño de la herramienta y retroalimentación continúa. De manera paralela el equipo de modelación realiza un ejercicio de análisis del funcionamiento de la herramienta y planteamiento de escenarios.

\section{Resultados}

En esta sección son presentados los principales avances de la modelación integrada. En la Figura 6 se muestra el comportamiento de los niveles del agua en el municipio de Nechí. Estos permiten representar la mancha de inundación y a su vez se convierten en el principal insumo para el modelo integrado de agentes. El Modelo Digital de Elevación usado es el de la NASA [44], que tiene una resolución de $30 x 30 \mathrm{~m}$. Los lugares por donde ha fallado el dique y la entrada del agua al pueblo son las condiciones de contorno y se usan los datos del nivel del agua (Nuevo Mundo: $3 m$, Pedro Ignacio: $1,5-2 m$, Santa Anita: $1,5-2 m$ y muralla: $0,5-1 m$ ). Estos puntos y niveles de agua fueron tomados a partir de una visita de campo. Adicionalmente para los limites no predefinidos como condición de contorno en el área activa, el programa impone una condición de no flujo. La condición inicial es seca, es decir que en todo el dominio no hay agua. Es elegida una rugosidad de 0,03 en el número de Manning. Respecto a los parámetros numéricos, se determinó un tamaño de paso de tiempo $\Delta t=2 s$, con el cual se garantiza un CFL no mayor a 1.5 , lo cual garantiza la estabilidad del modelo numérico y evita la presencia de oscilaciones indeseadas. El tiempo de simulación es de 24 horas con el fin de observar la mancha de inundación.
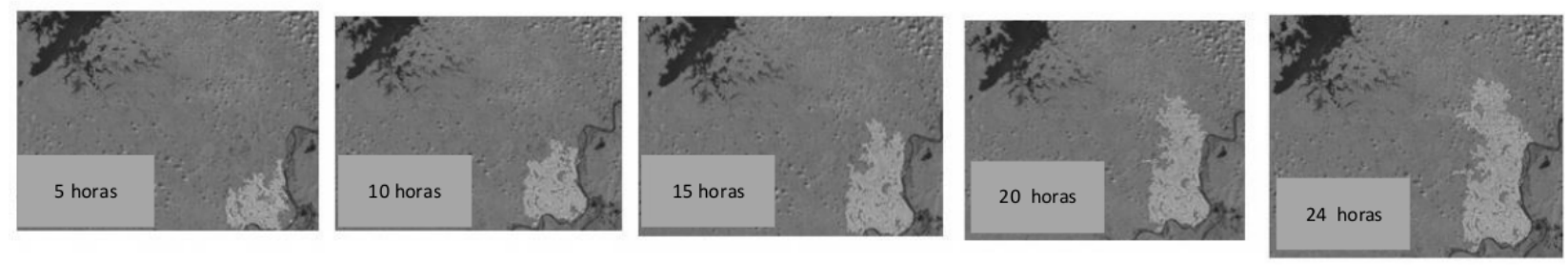

Figura 6. Modelación hidrodinámica. Niveles del agua-Mancha de inundación.

A partir de estos resultados, se observa cómo el flujo se propaga hacía la margen derecha del río y empieza su tránsito al interior de la ecorregión de la Mojana. Lo que afecta principalmente la población asentada en el área aferente al río Cauca. En la Figura 7 se observa la variación de la superficie libre del agua, en los puntos que ha fallado el dique. Estos niveles representan la dinámica del río que varía entre 31-36 m, evidenciando la amenaza por inundación. Este tipo de información es útil para: (i) la generación de un sistema de alertas tempranas, (ii) ubicación de asentamientos y el (iii) manejo de actividades productivas, entre otras.

La modelación de agentes tiene como propósito generar interacciones entre la población, la mancha de inundación y el uso del suelo. De esta manera se pretende analizar: (i) la relación de los niveles de inundación con el cambio en el sector productivo de la población, (ii) el análisis de zonas de riesgo debido a los procesos de amenaza por inundación y (iii) el análisis de vulnerabilidad territorial de la población frente a riesgos naturales, teniendo en cuenta aspectos sociales y económicos. A partir de estos aspectos, resulta importante generar escenarios de intervención, 


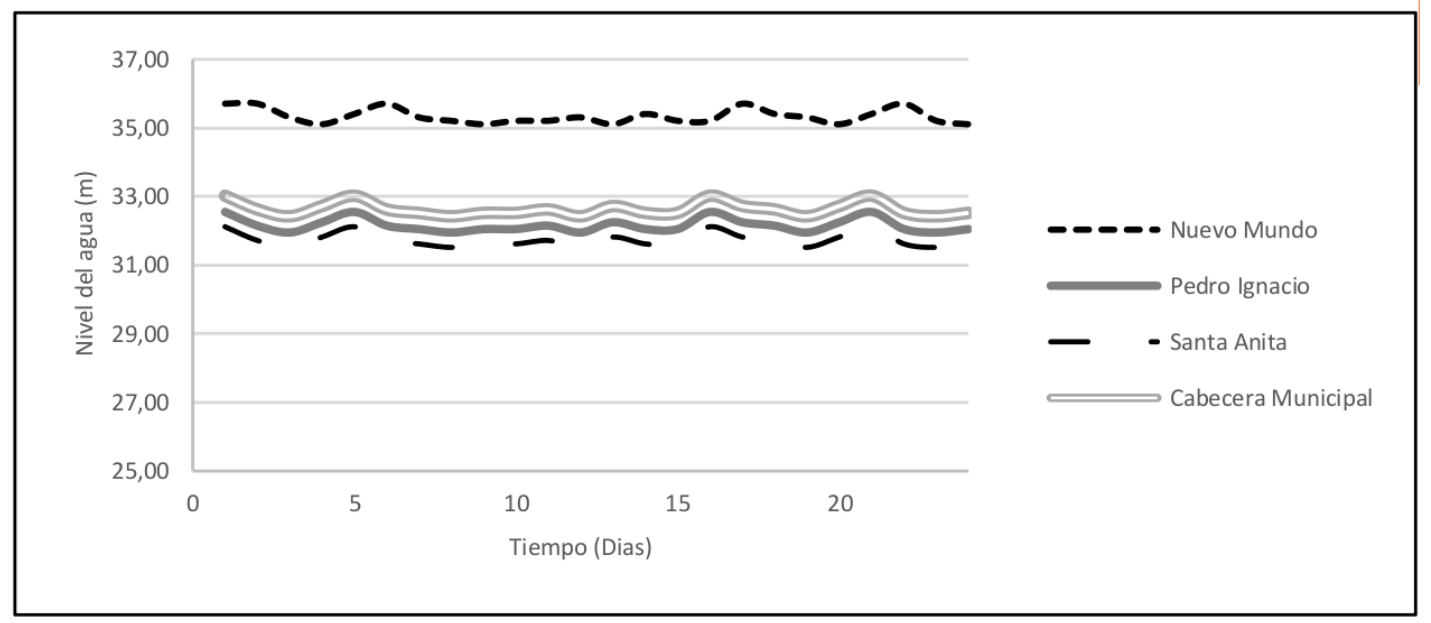

Figura 7. Variación de la superficie libre del agua.

que permitan apoyar los procesos de toma de decisiones en el municipio. La modelación integrada logró visualizar el comportamiento de la población y los recursos naturales en un territorio, lo que sin duda puede contribuir al diseño de políticas y la educación de la comunidad. En la Figura 8 es presentada una primera aproximación a la modelación con agentes.

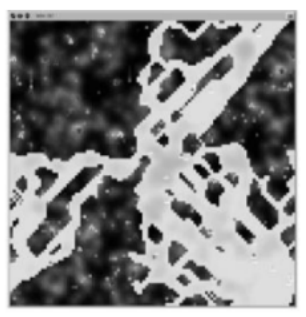

(a)

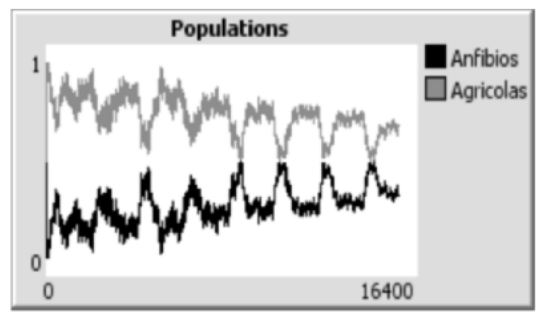

(b)

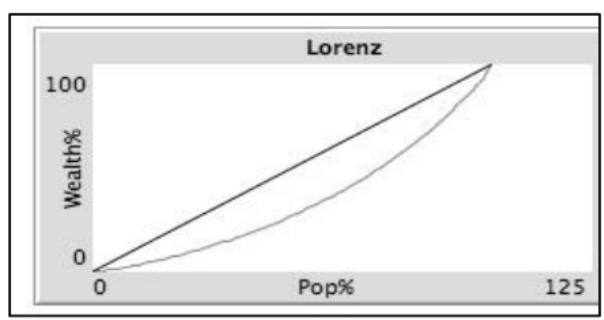

(c)

Figura 8. Modelación con agentes. (a) Representación del suelo, inundación y población. (b) Tamaño de poblaciones en el tiempo. Anfibios: población que se adapta a los procesos de inundación. Agrícolas: población que se adapta a las acondiciones secas. Se observa la variación de las poblaciones en función de los periodos de inundación. (d) Diagrama con el área comprendida entre la curva de Lorenz y la bisectriz del cuadrado, proporcional al coeficiente de Gini [32].

Allí se observa el territorio de la Mojana con sus características representadas en el uso del uso. La mancha de inundación reflejada en los niveles de agua y las interacciones de los agentes con este territorio. También son representados los asentamientos y los niveles de vulnerabilidad, destacados por tener diferentes tonalidades. Para esta primera simulación se hizo un análisis de reglas en el marco de la teoría evolutiva [32]. Dado que las reglas son preestablecidas y no tienen la posibilidad de ser modificadas desde los agentes, se puede manifestar que no se percibe variación desde el nivel macro [47]. Sin embargo, a partir de los frecuentes cambios de estado del sistema que ocurren en el nivel micro, es posible apreciar que en el nivel meso (i.e. razas, clases sociales y entornos) se configura una variedad de tendencias en cuanto a velocidad, asentamiento, crecimiento y adaptabilidad. En el nivel micro es más evidente la presencia y la influencia de la variación; la diversidad se manifiesta en el hecho que la población inicial de los agentes está dividida en dos razas: agrícolas y anfibios que a su vez pueden adoptar dos roles diferentes, el de búsqueda y el de asentamiento. Los sistemas selectivos según Campbell [51] eliminan o propagan variaciones específicas. Respecto a 
los selectores, se pueden identificar: sobrevivencia de grupos sociales, difusión selectiva entre grupos sociales, propagación de variaciones temporales y selección racional. Según Campbell [51] es notorio que las tradiciones se vean cada día más retenidas en el patrimonio material más que en la memoria oral-cultural. Este se ve reflejado en artefactos que perturban los procesos de aprendizaje de los individuos tanto como el ambiente natural. Además, Dopfer sugiere que el conocimiento no solo puede ser transportado por los agentes económicos sino también por los artefactos culturales que estos producen. Este sistema de retención se puede observar en diversos ambientes. En el caso de la Mojana, predomina una economía que gira en torno a la ganadería extensiva dando lugar a una cultura centrada en esta labor y desplazando prácticas culturales antiguas centradas en el agua [32]. La cultura ganadera extensiva se manifiesta materialmente a través de un paisaje poblado de artefactos para la crianza bovina, por ejemplo corrales, cercas, caminos y bebederos entre otros [32].

\section{Discusión y conclusiones}

En esta investigación se presenta una propuesta para la modelación integrada de un sistema socioecológico complejo, a partir de la modelación hidrodinámica y de los sistemas de agentes. Adicionalmente, son expuestos los avances de un ejercicio de modelación realizado en la ecorregión de la Mojana, municipio de Nechí.

Se pudo establecer que la herramienta ISIS 2D arroja los niveles de inundación del río y la velocidad que permiten representar la mancha de inundación. Así mismo los sistemas de agentes, desarrollados en NetLogo, evidencian que esta herramienta resulta ser útil para la generación de escenarios en el marco de la planeación, al representar las interacciones entre los agentes y los recursos naturales.

En los últimos años, los trabajos de modelación del territorio han ido aumentando [28], debido a que en algunas situaciones los modelos convencionales, o las herramientas empleadas, no logran llegar al nivel de detalle requerido y tampoco integran diferentes técnicas de modelación, y el conocimiento de los expertos. Con los procesos de modelación integrada se busca abordar el problema de la modelación desde una perspectiva que contemple escenarios de modelación espacio-temporal, donde puedan participar diseñadores y actores interesados.

Este estudio permitió evidenciar la potencialidad que tienen los métodos numéricos para la simulación del comportamiento de un fluido. No obstante, es necesario realizar ejercicios de refinamiento de mallas y variación de condiciones espaciales y temporales con el fin de generar mayores escenarios de simulación y analizar la estabilidad de las soluciones. Los modelos de simulación son un punto de partida en la toma de decisiones, pero no el único insumo. Desde una perspectiva interdisciplinar es importante contar con este tipo de herramientas para generar escenarios de intervención, pero se recomienda validar con las comunidades asentadas en el lugar e incluirlas en dichos procesos. Por esto la importancia de la modelación participativa.

Teniendo en cuenta el carácter complejo de las dinámicas en la Mojana, fue fundamental el trabajo de campo y la participación de las comunidades en el proceso de diseño del modelo. Las variables 
a modelar, las condiciones del modelo hidrodinámico y los procesos, fueron seleccionados con el apoyo de expertos locales. Se espera que, a futuro, se puedan generar procesos de modelación participativa para la selección de otras variables, el análisis de las escalas, la generación de escenarios y diferentes temáticas a modelar.

Adicionalmente este tipo de investigaciones contribuye al fortalecimiento del Sistema Nacional de Gestión del Riesgo (Ley 1523 de 2012-Colombia), en la medida que aporta a su conocimiento. Las herramientas de simulación como ISIS 2D y la simulación con agentes pueden generar escenarios para la toma de decisiones frente a alternativas como el manejo de los caños, la construcción de infraestructura y el fortalecimiento de los procesos de educación.

En los modelos integrados es importante el uso de diferentes escalas, resoluciones espaciales y temporales. De tal manera que para la modelación dinámica de un río se puede usar un modelo de espacio continuo y puede estar vinculado a un modelo ecológico, utilizando un modelo espacial regional. Según Letcher et al., (2013) [25] la conceptualización de las interacciones, la elección de la agregación o desagregación es subjetiva y es probable que afecte los resultados del modelo. De tal manera que el modelo puede ser rediseñado variando las escalas espacio-temporales. En este sentido, como trabajo futuro para el caso del territorio de la Mojana, se desean explorar diferentes escalas espacio-temporales enmarcadas en el propósito de la modelación y en la definición de los procesos a modelar.

\section{Referencias}

[1] L. Parrott, "Hybrid modelling of complex ecological systems for decision support: Recent successes and future perspectives". Ecological Informatics, 6(1), 2011, pp. 44-49. [En línea] Disponible en: http://doi.org/10.1016/j.ecoinf.2010.07.001

[2] DNP and FAO, Programa de desarrollo sostenible de la región de la Mojana, Colombia. I. G. Comunicaciones, Ed., (Primera ed). Bogotá D.C., 2003.

[3] G. Harris \& G. Harris, Seeking sustainability in an age of complexity. Seeking Sustainability in an Age of Complexity, 2007, [En línea] Disponible en: http://doi.org/10.1017/CBO9780511815140

[4] S. A. Levin, (1998). Ecosystems and the Biosphere as Complex Adaptive Systems. Ecosystems, 1(5), $431-436$. http://doi.org/10.1007/s100219900037.

[5] L. Parrott, (2002). Complexity and the Limits of Ecological Engineering. American Society of Agricultural Engineers I, 45(5), 1-6.

[6] D. A. Meyer, (1997). Towards the global: complexity, topology and chaos in modelling, simulation and computation 1 Introduction 2 Hierarchical e ciency 3 Topology induces complexity, (September), 0-5.

[7] Dawn C. Parker, Berger Thomas and S. M. M. (2002). Agent-Based Models of Land-Use and Land-Cover Change. LUCC Report Series, (6), 140.

[8] F. Bousquet and C. Le Page, (2004). Multi-agent simulations and ecosystem management: a review. Ecological Modelling, 176(3-4), 313-332. http://doi.org/10.1016/j.ecolmodel.2004.01.011.

[9] R. B., Matthews, N. G. Gilbert, A. Roach, J. G. Polhill and N. M. Gotts, (N.d.). Agentbased land-use models: a review of applications. LANDSCAPE ECOLOGY. Retrieved from http://search.ebscohost.com/login.aspx?direct=true \&db=edswsc \&AN=000250632100005\&lang=es\&site=edslive.

[10] L. An, (2012). Modeling human decisions in coupled human and natural systems: Review of agent-based models. Ecological Modelling, 229, 25-36. http://doi.org/10.1016/j.ecolmodel.2011.07.010

[11] S. Balbi, C. Giupponi, P. Pérez, and M. Alberti, (2013). A spatial agent-based model for assessing strategies of adaptation to climate and tourism demand changes in an alpine tourism destination. Environmental Modelling and Software, 45, 29-51. http://doi.org/10.1016/j.envsoft.2012.10.004. 
[12] D. Brown, S. Page, R. Riolo and W. Rand, (2004). Agent-based and analytical modeling to evaluate the effectiveness of greenbelts. Environmental Modelling \& Software, 19(12), 1097-1109. http://doi.org/10.1016/j.envsoft.2003.11.012.

[13] O. Barreteau and F. Bousquet, (2000). SHADOC: a multi-agent model to tackle viability of irrigated systems. Annals of Operations Research, 94(1-4), 139-162

[14] P. Schreinemachers and T. Berger, "An agent-based simulation model of human-environment interactions in agricultural systems". Environmental Modelling and Software, 26(7), 2011, pp. 845-859, [En línea] Disponible en: http://doi.org/10.1016/j.envsoft.2011.02.004

[15] S. Balbi, A. Prado, P. Gallejones, C. P. Geevan, G. Pardo, E. Pérez-Miñana and F. Villa, "Modeling trade-offs among ecosystem services in agricultural production systems". Environmental Modelling \& Software, 72, 2015, pp. 314-326, [En línea] Disponible en: http://doi.org/10.1016/j.envsoft.2014.12.017

[16] B. Anselme, F. Bousquet, A. Lyet, M. Etienne, B. Fady, and C. Le Page, Modelling of spatial dynamics and biodiversity conservation on Lure mountain (France). Environmental Modelling \& Software, 25(11), 2010, pp. 1385-1398. [En línea] Disponible en: http://doi.org/10.1016/j.envsoft.2009.09.001

[17] P. Kroes, Technical Artefacts: Creations of Mind and Matter: A Philosophy of Engineering Design, Philosophy of Engineering and Technology 6, 2012, pp. 127- 161, [En línea] Disponible en: http://doi.org/10.1007/978-94-0073940-6

[18] H. Arendt, Trabajo. En La condición humana, 2007, pp. 157-191. Editorial: PAIDOS IBERICA. Barcelona.

[19] J. Epstein, "Why model? Journal of Artificial Societies and Social, 11(4), 2008, p. 6. [En línea] Disponible en: http://doi.org/10.1080/01969720490426803

[20] M. Schwaninger, "Model-based management (MBM): a vital prerequisite for organizational viability". Kybernetes, 43(6), 2010, pp. 1419 - 1428.

[21] G. Restrepo, Aproximación Cultural al Concepto de Territorio. Revista Perspectiva Geográfica, 3, 2010, [En línea] Disponible en: http://datateca.unad.edu.co/contenidos/100007/Leturas_apoyo_Act6/Aproximación_cultural_al_concepto_de_terri torio_banrepcultural.org.pdf

[22] R. Barthel, S. Janisch, N. Schwarz, A. Trifkovic, D. Nickel, C. Schulz and W. Mauser, "An integrated modelling framework for simulating regional-scale actor responses to global change in the water domain". Environmental Modelling \& Software, 23(9), 2008, pp. 1095-1121, [En línea] Disponible en: http://doi.org/10.1016/j.envsoft.2008.02.004

[23] CEPAL, Población y Desarrollo. Modelos demográficos para la proyección de la demanda del sector social, 2007. Editorial CEPAL. Santiago de Chile.

[24] M. Schwaninger and M. Janovjak, Institut fur Betriebswirtschaft (Eds.), "Model-Based Management: A Systemic Approach from the Pharmaceutical Industry". Diskussionsbeitrage, 56. St. Gallen, 2008.

[25] R. A. Kelly, A. J. Jakeman, O. Barreteau, M. E. Borsuk, S. ElSawah, S. H. Hamilton and A. A. Voinov, "Selecting among five common modelling approaches for integrated environmental assessment and management". Environmental Modelling \& Software, 47, 2013, pp. 159-181. [En línea] Disponible en: http://doi.org/10.1016/j.envsoft.2013.05.005

[26] A. Voinov and F. Bousquet, "Modelling with stakeholders". Environmental Modelling \& Software, 25(11), 2010, pp. 1268-1281. http://doi.org/10.1016/j.envsoft.2010.03.007

[27] M. A. Max-Neef, Desarrollo a escala humana, 1993. Editorial Nordan-Comunidad. Montevideo, Uruguay.

[28] T. Berger, R. Birner, N. Mccarthy, J. DíAz and H. Wittmer, "Capturing the complexity of water uses and water users within a multi-agent framework". Water Resources Management, 21(1), 2006, pp. 129-148. [En línea] Disponible en: http://doi.org/10.1007/s11269-006-9045-z

[29] J. C. Refsgaard, J. P., van der Sluijs, A. L., Hojberg \& P. A. Vanrolleghem, "Uncertainty in the environmental modelling process - A framework and guidance". Environmental Modelling \& Software, 22(11), 2007, pp. 1543-1556. [En línea] Disponible en: http://doi.org/10.1016/j.envsoft.2007.02.004

[30] CORPOICA, El plan de la Mojana: aplicación del nuevo paradigma de desarrollo sostenible. CORPOICA, Vol 2, 1998.

[31] L. Posada, "Si no se concluyen las obras en La Mojana, el desastre será peor". 2012, UN periódico.

[32] P. A. Villegas, M. Melgarejo, and E. Pérez, MOJANA: Modelo Organizacional Jerárquico de Agentes Naturales del Agua. En proceso de publicación.

[33] Triviño, N., Escobar, J.A., and Villegas, P.A. Modelación numérica bidimensional de escenarios de inundación en el municipio de Nechí Antioquia. Proyecto de grado para optar al título de Ingeniera Civil. Facultad de Ingeniería. 
Pontificia Universidad Javeriana, 2015.

[34] U. Wilensky, (1998). NetLogo Wealth Distribution model. Center for Connected Learning and Computer-Based Modeling, Northwestern University, Evanston, IL. [En línea] Disponible en: http://ccl.northwestern.edu/NetLogo/models/WealthDistribution

[35] M. Felsen and U. Wilensky, NetLogo Urban Suite - Sprawl Effect model, Center for Connected Learning and Computer-Based Modeling, Northwestern University, Evanston, IL, 2007, [En línea] Disponible en: http://ccl.northwestern.edu/NetLogo/models/UrbanSuite-SprawlEffect

[36] G. Dunham, S. Tisue and U. Wilensky, NetLogo Erosion model. Center for Connected Learning and Computer-Based Modeling, Northwestern University, Evanston, IL, 2004, [En línea] Disponible en: http://ccl.northwestern.edu/NetLogo/models/Erosion

[37] ISIS, User Manual, ISIS 2D Cost effective, integrated software solutions. CHM2HILL, 2014.

[38] D. Wirasaet, E. J Kubatko, C. E Michoski, S. Tanaka, J. J Westerink and C, Dawson. "Discontinuos Galerkin methods whith nodal and hybrid modal/nodal triangular, quadrilateral and polygonal elements for nonlinear shallow water flow". Computer methods in applied mechanics and engineering, 270, 2014, pp. 113- 149.

[39] P. Kundu and L. Cohen, Fluid Mechanics. California, Academic, 1990.

[40] Henderson-Sellers and P. Giorgini, Agent-Oriented Methodologies. Idea group Inc. pp. 406. 2005.

[41] T. Filatova, P. H. Verburg, D. C. Parker, and C. A. Stannard, "Spatial agent-based models for socio-ecological systems: Challenges and prospects". Environmental Modelling \& Software, 45, 2013, pp. 1-7. [En línea] Disponible en: http://doi.org/10.1016/j.envsoft.2013.03.017

[42] O. P. R., Van, M. S. Krol, A. Hoekstra and R. R. Taddei, "Feedback mechanisms between water availability and water use in a semi-arid river basin: A spatially explicit multi-agent simulation approach". Environmental Modelling \& Software, 25, 2010, pp. 433-443.

[43] Z. Sun and D. Muller, "A framework for modeling payments for ecosystem services with agent-based models, Bayesian belief networks and opinion dynamics models". Environmental Modelling \& Software, 45, 2013, pp. $15-28$.

[44] USGS, Science for changing world, 2015, [En línea] Disponible en: http://glovis.usgs.gov

[45] G. Remolina, Del "BIG BANG" de las ciencias a su integración en el pensamiento complejo, 2014. Bogotá.

[46] J. A. Amozurrutia, Complejidad y ciencias sociales. Un modelo adaptativo para la investigación interdisciplinaria (Primera ed), 2011, [En línea] Disponible en: http://www.ceiich.unam.mx/0/51LibFic.php?tblLibros_id=449

[47] K. Dopfer, Evolutionary economics: a theoretical framework. The Evolutionary Foundations of Economics, 2005, [En línea] Disponible en: http://doi.org/http://dx.doi.org/10.1017/CBO9780511492297

[48] C. Olaya, "Models that Include Cows: The Significance of Operational Thinking". 30th International Conference on System Dynamics Society, 2012, pp. 1-19.

[49] M. Aguilera, La Mojana: riqueza natural y potencial económico. Documentos de trabajo sobre economía regional, 2004. Editorial Banco de la República. Cartagena.

[50] A. Serna, Memorias en crisoles propuestas teóricas, metodológicas y estratégicas para los estudios de memoria. IPAZUD, Instituto para la Pedagogía, Bogotá, D.C., Ed. Igarss 2014, [En línea] Disponible en: http://doi.org/10.1007/s13398-014-0173-7.2.

[51] D.T Campbell, Variation and Selective Retention in Sociocultural Evolution in Social Change in Developing Areas: A Reinterpretation of Evolutionary Theory, H.R. Barringer, G.I. Blanksten, and R.W. Mack, Editors. 1965, Schenkman: Cambridge, Mass. p. 19-49

[52] Rodríguez, C. (2014). Modelamiento de estaciones TransMilenio mediante Autómatas Celulares: lecciones aprendidas. En: Ingeniería, Vol. 19, No. 2, pp. 105-113.

\section{Paula Andrea Villegas González}

Institución, Pontificia Universidad Javeriana; afiliación: Instituto Geofísico; Facultad de Ingeniería, estudiante Doctorado en Ingeniería Pontificia Universidad Javeriana; docente Investigadora Universidad Católica de Colombia; magister en Hidrosistemas Pontificia Universidad Javeriana; ingeniera Civil Universidad Nacional de Colombia. Correo electrónico: pvillegas@javeriana.edu.co

\section{Nathaly Triviño León}

Institución, Pontificia Universidad Javeriana; afiliación: Facultad de Ingeniería, estudiante Maestría en Hidrosistemas 
Pontificia Universidad Javeriana; ingeniera Civil Pontificia Universidad Javeriana. Correo electrónico: ntrivino@ javeriana.edu.co

\section{Jorge Alberto Escobar Vargas}

Institución, Pontificia Universidad Javeriana; afiliación, Facultad de Ingeniería; docente, Pontificia Universidad Javeriana; doctor Mecánica de Fluidos Computacional University Cornell; magister en Métodos Numéricos para el Cálculo y Diseño en Ingeniería, Universidad Politécnica de Cataluña; magíster en Hidrosistemas Pontificia Universidad Javeriana; magíster en Mecánica de Fluidos Ambiental, Cornell University; ingeniero Civil, Universidad Nacional. Correo electrónico: jorge-escobar@javeriana.edu.co

\section{Nelson Obregón Neira}

Institución, Pontificia Universidad Javeriana; afiliación, Director Instituto Geofísico, Facultad de Ingeniería; docente, Pontificia Universidad Javeriana; postdoctorado University of California Santa Barbara; doctorado en Hydrologic Sciencies University of California Santa Barbara; magíster en Ingeniería Civil Universidad de los Andes; Ingeniero Civil, Universidad Francisco de Paula Santander. Correo electrónico: nobregon@javeriana.edu.co

\section{Mauricio González Méndez}

Institución, Universidad Católica de Colombia; afiliación, Facultad de Ingeniería, Docente Universidad Católica de Colombia; estudiante de Doctorado en Ingeniería Pontificia Universidad Javeriana; magíster en Ingeniería Ambiental Universidad de los Andes; ingeniero Civil Universidad de los Andes. Correo electrónico: amgonzalez@ ucatolica.edu.co

\section{Ramón Eduardo González Salazar}

Institución, Pontificia Universidad Javeriana; afiliación, Facultad de Teología Pontificia Universidad Javeriana, docente; magíster en Estudios Políticos, Pontificia Universidad Javeriana; licenciado en filosofía, Universidad Santo Tomas. Correo electrónico: regonzal@javeriana.edu.co

\section{María Juliana Flórez Flórez}

Institución, Pontificia Universidad Javeriana; afiliación, Instituto Pensar Pontificia Universidad Javeriana, docente; doctora en Psicología Social, Universitat Autónoma de Barcelona; maestría en Psicología Social, Universitat Autónoma de Barcelona; especialización en Cooperación y Desarrollo; licenciada en Psicología. Correo electrónico: florez.maria@javeriana.edu.co 